\title{
A CYTOPLASMICALLY CONTROLLED ABNORMALITY OF THE TETRADS OF COPRINUS LAGOPUS
}

\author{
P. R. DAY \\ John Innes Horticultural Institution, \\ Bayfordbury, Hertford, Herts
}

Received 30.v. $5^{8}$

Among the gill-bearing forms of the higher basidiomycetes (Agaricacæ) meiosis in the basidium is generally followed by the production of four haploid basidiospores in a tetrad. Some Agaricacæ have two-spored basidia such as the two-spored forms of species of the genus Mycena (Smith, 1934) or the cultivated mushroom Psalliota campestris. Examples of basidia bearing one, three, five, six or more basidiospores appear to be less common but they have been reported in Psalliota campestris (Kligman, I943) and Coprinus species (Buller, I922, I931, and Sass, 1932).

Tetrads with more or less than the normal number of four spores were found on the gills of some fruit bodies from crosses of two wildtype monocaryons of Coprinus lagopus (Day, 1957). This paper describes an investigation of this abnormality.

\section{METHODS}

(i) Media

Monocaryons and dicaryons were grown on a complete medium made up from Fries (1953) medium: L-asparagine, $2 \cdot 0$ g.; $\mathrm{NH}_{4}$ tartrate, $\mathrm{I} \cdot 5 \mathrm{~g}$.; $\mathrm{KH}_{2} \mathrm{PO}_{4}$, I.o g. ; $\mathrm{Na}_{2} \mathrm{HPO}_{4}, 2 \cdot 25 \mathrm{~g}$.; $\mathrm{Na}_{2} \mathrm{SO}_{4}, 0 \cdot 29$ g. ; thiamin, $40 \mu \mathrm{g}$.; glucose, $20 \cdot 0 \mathrm{~g}$. ; agar, $20 \mathrm{~g}$.; water to rooo ml. supplemented with Oxoid yeast extract, $0 \cdot 75 \mathrm{~g}$.; bacteriological peptone (Eupepton No. 2, supplied by Messrs Allen \& Hanbury, Ware, Herts), $\mathrm{o} \cdot 75 \mathrm{~g}$. and Oxoid malt extract, $0 \cdot 6 \mathrm{~g}$.

Fruit bodies were produced on horse dung which had been moistened and autoclaved for half an hour in half-pint milk bottles closed with aluminium foil. They appeared from 7-1o days after inoculation with a dicaryon.

All cultures were incubated at $28^{\circ} \mathrm{C}$. in daylight, in a glass-fronted incubator.

\section{(ii) Stocks}

Two basidiospores were isolated by Professor D. Lewis from a single fruit body occurring on a manure heap at Barwick Ford, Herts, in 1952. The cultures they gave rise to were numbered 54 and 68 and since they were compatible they were given the mating types $A_{1} B_{2}$ and $A_{2} B_{1}$ respectively.

Stock cultures were maintained under mineral oil at room temperature and at $4^{\circ} \mathrm{C}$.

\section{(iii) Crosses}

When two haploid, compatible monocaryons are crossed inocula of each are placed 2-4 mm. apart on complete medium in a petri dish. After 2-3 days' incubation both monocaryons may become dicaryotised, when the vigorous hyphæ with clamp connections can easily be recognised. In describing a cross, the monocaryon which is the source of the dicaryon is written first although, of course, dicaryotic mycelia can be taken from either parent. 


\section{(iv) Cytology}

To study meiosis in the basidia fruit bodies at various stages of development were fixed in $\mathrm{I}: 3$ acetic alcohol for half an hour and stored in 70 per cent. ( $\mathrm{v} / \mathrm{v}$ ) alcohol at $4^{\circ} \mathrm{C}$. Fragments of gill tissue were transferred directly to cold IN HCI for 5 minutes and then to IN $\mathrm{HCI}$ at $60^{\circ} \mathrm{C}$. for 5 to 7 minutes. After rinsing in distilled water the fragments were tapped out in a drop of a freshly-diluted and filtered solution of synthetic orcein ( $1 \mathrm{ml} .5$ per cent. $(\mathrm{w} / \mathrm{v}$ ) stock solution in glacial acetic acid $+\mathrm{I} \mathrm{ml}$. distilled water). Pressure was applied through two layers of filter paper to a cover slip placed over the drop and, after heating to improve differential staining, each slide was sealed with paraffin wax and stored at $4^{\circ} \mathrm{C}$.

Other methods are described where relevant in the text.

\section{RESULTS}

In January $\mathrm{I} 957$ the dicaryon $68\left(\mathrm{~A}_{2} \mathrm{~B}_{1}\right) \times 54\left(\mathrm{~A}_{1} \mathrm{~B}_{2}\right)$ gave rise to normal fruit bodies. The gill surfaces of a normal fruit body are black with densely-crowded basidiospores in tetrads. Before maturity the gills are white and there is a stage when each fully-formed basidiospore is colourless. However, as it reaches maturity, the deposition of a black pigment begins at the end of the spore distal to the sterigma and continues until the entire spore wall is darkly pigmented. The density of tetrads is about 3000 per sq. $\mathrm{mm}$. of gill surface (plate, fig. 2).

At the same time the reciprocal dicaryon $54\left(\mathrm{~A}_{1} \mathrm{~B}_{2}\right) \times 68\left(\mathrm{~A}_{2} \mathrm{~B}_{1}\right)$ gave rise to abnormal fruit bodies with pale almost white gills at maturity (plate, fig. I). The tetrad density on the gills of these fruit bodies ranged from o-6oo per sq. mm., the denser areas occurring in patches. Among the mature tetrads were basidia arrested at various stages of development : some were completely sterile, others produced small sterigmata or spore rudiments. Some of the mature "tetrads" were abnormal, having two, three, five or six spores (plate, figs. 3 and 4 ). One basidium was seen with eight spores. The distribution of these abnormal tetrads was non-random. Certain areas of a gill produced mainly threes and fours and other areas mainly fours and fives. Abnormal tetrads occasionally occurred in pairs. Estimates of the relative frequencies of the different types of tetrad were made by counting 2000 tetrads of a typical pale fruit body. These are given in table $\mathrm{I}$. The relative frequencies varied between fruit bodies and between gills within a fruit body. Thus, although the average frequency of abnormal tetrads from table I is 12.9 per cent., patches of the gills of some pale fruit bodies had frequencies as high as 40 per cent.

Twenty-five abnormal tetrads were isolated with a micromanipulator and transferred to complete medium. In no case did all the spores of a tetrad germinate, whereas up to 75 per cent. of tetrads from normal fruit bodies germinate completely. The germination results are given in table $\mathrm{I}$. Two pentads which each gave rise to four colonies are of special interest. The colonies were tested for mating type with tester stocks $\mathrm{C}_{527}\left(\mathrm{~A}_{3} \mathrm{~B}_{1}\right)$ and $\mathrm{C}_{728}\left(\mathrm{~A}_{2} \mathrm{~B}_{4}\right)$. The first set of four was 
parental ditype for $A$ and $B$ alleles $\left(2 A_{1} B_{2}: 2 A_{2} B_{1}\right)$, the second set was tetratype except that one class $\left(A_{1} B_{2}\right)$ was missing and another $\left(A_{1} B_{1}\right)$ was represented twice $\left(2 A_{1} B_{1}: A_{2} B_{1}: A_{2} B_{2}\right)$. Among the

TABLE I

Abnormal tetrad frequencies and spore germination of a pale fruit body of the dicaryon $54\left(A_{1} B_{2}\right) \times 68\left(A_{2} B_{1}\right)$

\begin{tabular}{|c|c|c|c|}
\hline Tetrad spore no. & Per cent. & $\begin{array}{l}\text { No. of tetrads } \\
\text { isolated }\end{array}$ & $\begin{array}{l}\text { Spores germinated } \\
\text { per tetrad }\end{array}$ \\
\hline 6 & 0.2 & I & o \\
\hline 5 & $4 \cdot 5$ & $\left\{\begin{array}{r}2 \\
13\end{array}\right.$ & $\begin{array}{l}4^{*} \\
3 \text { or less }\end{array}$ \\
\hline 4 & $87 \cdot I$ & 0 &. \\
\hline 3 & $7 \cdot 9$ & $\left\{\begin{array}{l}3 \\
4\end{array}\right.$ & $\begin{array}{l}2 \\
1\end{array}$ \\
\hline & & 1 & 0 \\
\hline 2 & 0.3 & I & I \\
\hline
\end{tabular}

* Mating types :- $2 \mathrm{~A}_{1} \mathrm{~B}_{1}, 2 \mathrm{~A}_{2} \mathrm{~B}_{2}$ (non-parental ditype). $2 A_{1} B_{1}, A_{2} B_{1}, A_{2} B_{2}$ (tetratype?).

other cultures from incomplete sets, dwarfed, gnarled mycelia were common.

Several dicaryons were produced by crossing compatible monocaryons with normal vigour derived from basidiospores produced by pale fruit bodies. All gave rise to normal fruit bodies.

TABLE 2

Distribution of nuclei and sterigmata in the basidia of an immature pale fruit body from the dicaryon $54 \times 68$

\begin{tabular}{|c|c|c|c|c|c|c|c|c|}
\hline \multirow{2}{*}{ Sterigmata } & \multicolumn{6}{|c|}{ Nuclei } & \multirow{2}{*}{ Total } & \multirow{2}{*}{ Per cent } \\
\hline & 2 & 3 & 4 & 5 & 6 & 7 & & \\
\hline 2 & I & o & 2 & o & o & I & 4 & $3 \cdot 2$ \\
\hline 3 & I & 6 & 7 & 4 & 2 & o & 20 & 16.0 \\
\hline 4 & 4 & 7 & 8I & 4 & 2 & I & 99 & $79 \cdot 2$ \\
\hline $\begin{array}{r}\text { Total } \\
\text { Per cent. }\end{array}$ & $\begin{array}{c}6 \\
4^{\cdot} \cdot 8\end{array}$ & $\begin{array}{l}13 \\
10.4\end{array}$ & $\begin{array}{l}9^{2} \\
73 \cdot 6\end{array}$ & $\begin{array}{c}8 \\
6 \cdot 4\end{array}$ & $\begin{array}{c}4 \\
3 \cdot 2\end{array}$ & $\begin{array}{c}2 \\
\mathrm{I} \cdot 6\end{array}$ & $\begin{array}{r}\mathrm{I} 25 \\
\ldots\end{array}$ & $\begin{array}{l}\ldots \\
\ldots\end{array}$ \\
\hline
\end{tabular}

Acetic-orcein preparations of gill fragments from young fruit bodies produced by the dicaryon $54 \times 68$ showed that meiosis in many basidia was delayed or arrested at Mr. Stages after Mr often showed bridges, chromosome fragments and micronuclei. At stages after $\mathrm{T}_{2}$ departures from the normal number of four nuclei were common. The number of nuclei and sterigmata for each of 125 basidia were scored in a preparation made from a fruit body with immature tetrads. The results are given in table 2. Eighty-one basidia $(64 \cdot 8$ per cent.) were normal with four sterigmata and four nuclei. 
Corner (1948) has suggested that the sterigmata and basidiospores arise independently of the nuclei and centrosomes of the basidium. If the sterigmata were organised by the nuclei there would be as many sterigmata as nuclei. There might be fewer sterigmata in abnormal fruit bodies because of nuclear unbalance brought about by aborted meiosis, but there could never be more sterigmata than nuclei. Table 2 shows that fourteen basidia (I I 2 per cent.) had more sterigmata than nuclei. Some of these could be explained by loss of nuclei through the sterigmata during the preparation of the slide. However, in five instances all the rudimentary spores were still attached.

The reciprocal crosses were repeated three months later on a larger scale using subcultures $54^{\prime}$ and $68^{\prime}$ of the original stocks but the differences were not as extreme as previously. The dicaryon $54^{\prime} \times 68^{\prime}$ gave pale fruit bodies but $68^{\prime} \times 54^{\prime}$ gave normal and pale fruit bodies. Some of the pale fruit bodies had small sectors of dark gills with normal tetrad densities and few abnormal tetrads. These were separated by larger sectors of pale gills with the usual patchy distribution of normal and abnormal tetrads (plate, fig. 6).

Subcultures of the original dicaryon $68 \times 54$ which had given normal fruit bodies gave pale as well as normal fruit bodies. These occurred together in the same flask and in one instance a fruit body of each type arose from adjacent centres. A dicaryon isolated from the stipe of the pale fruit body gave rise to pale fruit bodies when inoculated to dung. A similar dicaryon isolated from the stipe of the normal fruit body gave rise to normal fruit bodies.

Normal and pale dicaryons were resolved into their component monocaryons by using a method developed by Lewis (unpublished). A chlamydospore (plate, fig. 5) suspension is obtained by crushing aerial mycelium scraped from the surface of a Io-I4-day-old culture of a dicaryon, in sterile water. The suspension is filtered through glass wool to remove debris, plated and incubated for 24-36 hours. Many chlamydospore germ tubes and resulting hyphæ bear clamps but a proportion have no clamps. The hyphal tips without clamps are cut off to include 2-5 cells and transferred to slants when a variable proportion give rise to monocaryons. The nuclei of these cultures can be identified by mating tests with the two parental cultures.

Three dicaryons were resolved in this way. They were :

I. the stipe subculture of the original dicaryon $68 \times 54$ which gave normal fruit bodies,

2. the stipe subculture of the same dicaryon which gave pale fruit bodies,

3. $54^{\prime} \times 68^{\prime}$ giving pale and some sectored pale fruit bodies.

The resulting monocaryons were identified by mating tests with $54^{\prime}\left(\mathrm{A}_{1} \mathrm{~B}_{2}\right)$ and $68^{\prime}\left(\mathrm{A}_{2} \mathrm{~B}_{1}\right)$. Representative monocaryons from I and 2 were used in crosses and the resulting dicaryons inoculated to dung to determine fruit body type. The results are shown in table 3 . 


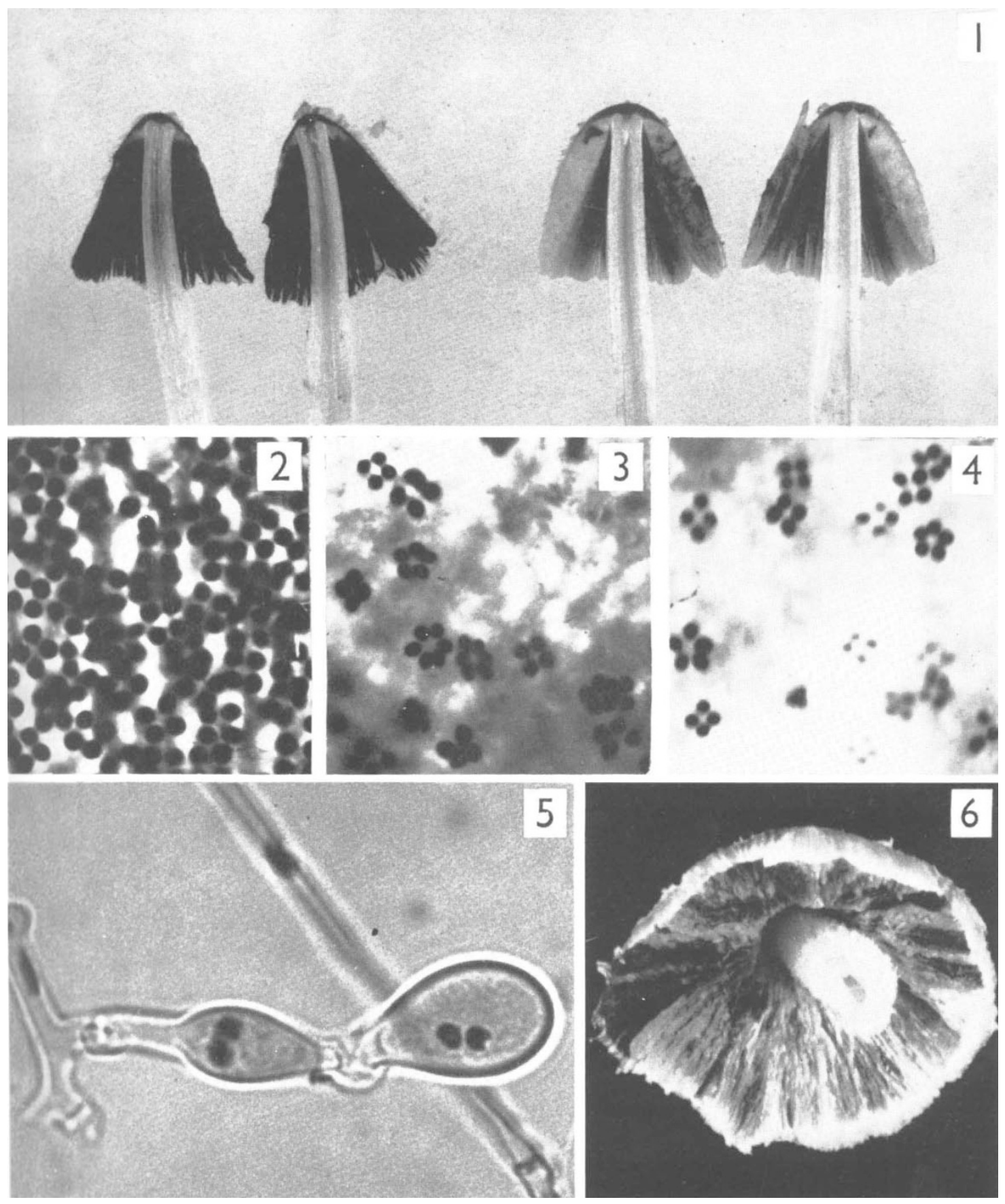

FIG. I.-Left ; Normal fruit body from $68 \times 54$.

Right ; Pale fruit body from $54 \times 68$.

Both fruit bodies split longitudinally. c. natural size.

FIG. 2.-Gill surface of normal fruit body. c. $\times 450$.

FIGs. 3 and 4.-Gill surfaces of pale fruit body-normal and abnormal tetrads. c. $\times 450$.

FIG. 5.-Chlamydospores of a dicaryon stained with acetic-orcein. c. $\times 2000$.

Frg. 6. - Sectored pale fruit body viewed from below. $\times 3$. 
Four of the eleven dicaryon cultures produced by single chlamydospores from $68 \times 54$ (normal) were fruited. Three gave pale fruit bodies and one gave normal fruit bodies. Eight dicaryons from $68 \times 54$ (pale) chlamydospores all gave rise to pale fruit bodies only.

TABLE 3

Results of resolving three dicaryons into their components

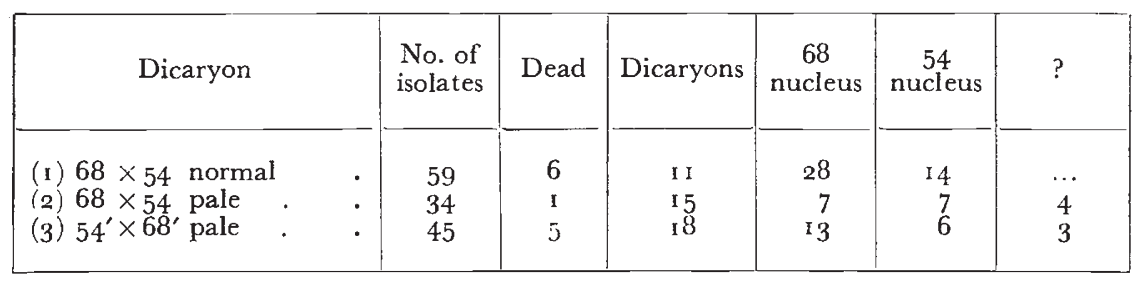

Fruit body types produced by crossing component monocaryons

\begin{tabular}{|c|l|}
\hline Cytoplasm parent & \multicolumn{1}{|c|}{ Fruit bodies } \\
\hline 54 (ex normal dicaryon) $\times 68^{\prime}$ & \\
54 (ex pale dicaryon) $\times 68^{\prime}$ & normal \\
68 (ex normal dicaryon) $\times 54$ (ex pale dicaryon) & pale (some sectored) \\
68 (ex pale dicaryon) $\times 54$ (ex pale dicaryon) & pale \\
68 (ex pale dicaryon) $\times 54$ (ex normal dicaryon) & pale \\
54 (ex pale dicaryon) $\times 68$ (ex normal dicaryon) & pale \\
\hline
\end{tabular}

\section{DISCUSSION}

When two compatible haploid monocaryons of Coprinus lagopus are crossed the two mycelia grow and, when they touch, exchange nuclei. The monocaryon growth of each inoculum gives way to dicaryon growth. Since reciprocal dicaryons contain the same nuclei, differences between them must reflect differences between the cytoplasms of the parental monocaryons. It is justifiable to assume that the monocaryon which receives the migratory nuclei will contribute the bulk of the cytoplasm to the resulting dicaryon.

The initial difference between the reciprocal dicaryons $68 \times 54$ and $54 \times 68$ suggests a cytoplasmic difference between the two stocks. The deterioration of the 68 monocaryon and the normal dicaryon suggest that the cytoplasmic change may be a result of ageing. The occurrence of both types of fruit bodies from a common inoculum and the occurrence of sectored fruit bodies demonstrate segregation of the cytoplasmic elements. The results from resolving dicaryons show that in the main the components follow the cytoplasmic character of the dicaryon parent. The exceptions are most likely due to variation between the samples of cytoplasm contained in chlamydospores from a common mycelium. The variation between four dicaryons which originated as chlamydospores of the dicaryon $68 \times 54$ (normal) is evidence of this. 
It seems likely that the primary effect of abnormal cytoplasm is on meiosis, leading to abnormal divisions in many basidia. The isolated tetrads show the reduced viability and stunted mycelia expected from chromosomal unbalance due to aborted meiosis. One tetrad points to premature mitosis as another effect. The unusual tetrad $2 A_{1} B_{1}, A_{1} B_{2}, A_{2} B_{2}$ from a set of five spores can be most easily explained by assuming that it was tetratype and that a mitotic division of the $A_{1} B_{1}$ nucleus took place in the basidium and the missing nucleus $\left(\mathrm{A}_{2} \mathrm{~B}_{1}\right)$ was lost in the spore that did not germinate. In a normal fruit body mitosis occurs after each nucleus has migrated into a basidiospore. Premature mitosis in the basidium would contribute to the classes in table 2 with more than four nuclei. A third effect of abnormal cytoplasm is that on the organisation of sterigmata and basidiospores. so that departures from the normal number are common.

In Coprinus sterquilinus (Sass, I935) aborted meiosis and abnormal tetrads have been brought about by overcrowding in the fruiting cultures. It seems unlikely that overcrowding is responsible for the abnormalities described here since single pale fruit bodies have been frequently seen in the culture flasks as well as fruit bodies of all three types ; normal, sectored and pale.

\section{SUMMARY}

Fruit bodies with pale gills bearing tetrads with from two to six basidiospores were produced by crossing two wild-type stocks. The reciprocal cross gave normal fruit bodies. When the parent stocks were subcultured and the crosses repeated three months later, both gave rise to abnormal fruit bodies although some from the cross which had previously been normal had normal sectors.

Normal and abnormal dicaryons were resolved into their components and these, when used as cytoplasm parents in further crosses, with some exceptions, followed their parent dicaryons in the type of fruit body produced.

A cytological examination of abnormal fruit bodies revealed. irregularities in the meiotic divisions in the basidia producing bridges and micronuclei. There was no correlation between the number of nuclei and sterigmata in abnormal basidia.

Acknowledgments.-I am indebted to Professor D. Lewis, F.R.s., for his helpful discussion and encouragement. I also wish to express my appreciation of Mrs. Margaret Lamdin's assistance.

\section{REFERENCES}

BULleR, A. H. R. 1922. Researches on Fungi. Longmans Green, London. Vol 2, p. 492.

BULLER, A. H. R. 1931. Researches on Fungi. Longmans Green, London. Vol. 4, p. 329 .

CORNER, E. J. H. 1948. Studies in the basidium. I. The ampoule effect, with a note on nomenclature. New Phytol., 47, 22-5I. 
DAY, P. R. I957. Abnormal tetrads in Coprinus lagopus. Abstr. Heredity, II, 426.

FRIES, L. I 953. Factors promoting growth of Coprinus fimetarius (L) under high temperature conditions. Physiol. Plant., 6, 551-563.

KLigman, A. м. I943. Some cultural and genetic problems in the cultivation of the mushroom, Agaricus campestris Fr. Amer. F. Bot., 30, 745-763.

SASs, J. E. 1932. The cytology of a diploid sterile hymenomycete. Mycologia, 24, 229-232.

SASs, J. E. I935. Cytological aspects of physiological sterility in Coprinus sterquilinus. Ann. Bot., 49, 151-154.

SMITH, A. H. I 934 . Investigations of two-spored forms in the genus, Mycena. Mycologia, 26, 305-331. 